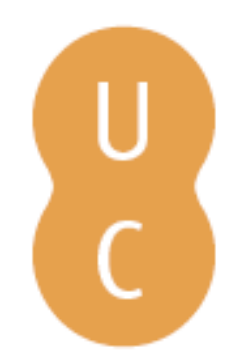

\title{
pombalina
}

\section{Émotion, cognition et immunité}
Autor(es):
Fillion, Use
Publicado por: Imprensa da Universidade de Coimbra; Les Éditions de L'IQRC (Les Presses de l'Université Laval)
URL
persistente:
URI:http://hdl.handle.net/10316.2/32641
DOI:
DOI:http://dx.doi.org/10.14195/978-989-26-0805-1_10
Accessed : $\quad$ 26-Apr-2023 10:47:34

A navegação consulta e descarregamento dos títulos inseridos nas Bibliotecas Digitais UC Digitalis, UC Pombalina e UC Impactum, pressupõem a aceitação plena e sem reservas dos Termos e Condições de Uso destas Bibliotecas Digitais, disponíveis em https://digitalis.uc.pt/pt-pt/termos.

Conforme exposto nos referidos Termos e Condições de Uso, o descarregamento de títulos de acesso restrito requer uma licença válida de autorização devendo o utilizador aceder ao(s) documento(s) a partir de um endereço de IP da instituição detentora da supramencionada licença.

Ao utilizador é apenas permitido o descarregamento para uso pessoal, pelo que o emprego do(s) título(s) descarregado(s) para outro fim, designadamente comercial, carece de autorização do respetivo autor ou editor da obra.

Na medida em que todas as obras da UC Digitalis se encontram protegidas pelo Código do Direito de Autor e Direitos Conexos e demais legislação aplicável, toda a cópia, parcial ou total, deste documento, nos casos em que é legalmente admitida, deverá conter ou fazer-se acompanhar por este aviso.

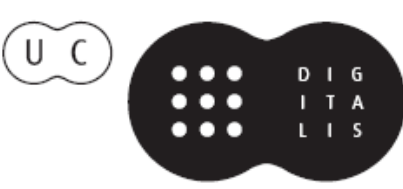


GILLES KIROUAC

Coordonnateur Scientifique

Cognition
et Émotions 
(Página deixada propositadamente em branco) 


\title{
GILLES KIROUAC
}

Coordonnateur Scientifique

\section{Cognition et Émotions}

\author{
AUTEURS \\ Klaus R. Scherer \& Janique Sangsue \\ Pierre Philippot \\ Dario Galati \\ Bernard Rimé \\ Pierre Gosselin \\ Ursula Hess \\ Arvid Kappas \& Jean Descôteaux \\ Pio E. Ricci Bitti \\ Pedro Luzes \\ Lise Fillion \\ Ramon Bayés \\ Coimbra - Imprensa da Universidade


COORDENAÇÃO EDITORIAL

Imprensa da Universidade de Coimbra

CONCEPÇÃO GRÁFICA

António Barros

PAGINAÇÃo

António Resende

[Universidade de Coimbra]

EXECUÇÃO GRÁFICA

G.C. - Gráfica de Coimbra, Lda.

Palheira - Assafarge - Apart. 3068

300I-453 Coimbra Codex

ISBN 972-8704-19-4

ISBN 2-7637-81।8-7

DEPÓSITO LEGAL 210430/04

(C) MAIO 2004, Imprensa DA UnIVERSIDAdE DE COIMBRA

\author{
OBRA PUBLICADA COM O PATROCINIO DO \\ INSTITUTO DE PSICOLOGIA COGNITIVA \\ FCT: FUNDAÇÃO PARA A CIÊNCIA E A TECNOLOGIA \\ MINISTÉRIO DA CIÊNCIA E DO ENSINO SUPERIOR \\ apolo do Programa Operacional CiênCIa, TeCnologia, Inovação \\ DO QUADRO COMUNITÁRIO DE APOIO
}


Lise Fillion

University of Miami

\title{
ÉMOTION, COGNITION ET IMMUNITÉ (*)
}

\begin{abstract}
RÉSUMÉ
La thèse d'un lien entre les émotions et la maladie est envisagée depuis longtemps. Qu'en est-il de sa démonstration scientifique? La psychoneuro-immunologie (PNI) propose un mécanisme permettant de soutenir cette thèse. Son but ultime est d'expliquer l'influence des facteurs psychosociaux sur la résistance des individus à la maladie. La plupart des travaux réalisés en $\mathrm{PNI}$ appartiennent au domaine du stress psychologique et empruntent un cadre théorique provenant des domaines de la psychologie sociale et de la psychophysiologie. Quelques études relèvent directement de la psychologie des émotions. En respectant une proposition de rapprocher les études sur le stress psychologique et celles portant plus spécifiquement sur les émotions, cette présentation vise à fournir une intégration de différentes recherches réalisées en PNI. Dans un premier temps, cette intégration fournit un cadre théorique et une démonstration empirique permettant de soutenir la thèse d'une relation entre l'émotion et l'altération du fonctionnement immunitaire. Suite à une brève présentation de la psychoneuro-immunologie et du concept stress-émotion, plusieurs études reliant les stresseurs, le stress-émotion et l'immunité sont ainsi présentées. Les résultats démontrent clairement une altération immunologique en réaction à différents types d'événements stressogènes, soit en milieu naturel ou en laboratoire. En plus des stresseurs aigus, la recherche a également démontré une modulation immunitaire chez des sujets exposés à des stresseurs chroniques. Quoique
\end{abstract}

${ }^{*}$ *) Cet écrit a été réalisé grâce au soutien financier du Conseil de recherches en sciences humaines du Canada, bourse post-doctorale 756-93-040I. 
l'immunomodulation ne soit pas toujours liée à la condition d'exposition, elle apparait souvent associée à l'intensité de la réponse stress-émotion. Les résultats soutiennent l'hypothèse d'un lien entre l'émotion et l'immunité. Dans un second temps, l'intégration des différents travaux vise à faire ressortir l'importance d'inclure des variables médiatrices, tels l'évaluation cognitive du stresseur et les modes de gestion, afin de mieux comprendre les différences individuelles observées dans les profils de réaction. Le présent travail se termine par l'illustration de la pertinence d'inclure l'évaluation cognitive dans les études en PNI.

\section{ÉMOTION, COGNITION ET IMMUNITÉ}

La psychoneuro-immunologie (PNI) est souvent définie comme une extension de la recherche sur le stress, car son but ultime est d'expliquer l'influence des facteurs psychosociaux sur la résistance des individus à la maladie. En respectant une proposition récente de rapprocher les études sur le stress et celles portant plus spécifiquement sur les émotions, une intégration de différentes recherches réalisées en PNI est effectuée. Ce travail de synthèse vise, dans un premier temps, à soutenir la thèse d'une relation entre l'exposition à un événement stressogène, un phénomène émotionnel et une altération du fonctionnement immunitaire. Dans un second temps, l'intégration des travaux fait ressortir la nécessité d'inclure des variables médiatrices, telles que certains facteurs cognitifs, afin de mieux comprendre les différences individuelles observées dans les profils de réaction. L'illustration de la pertinence d'inclure l'évaluation cognitive termine cette présentation.

190

\section{Considérations Préliminaires}

La première partie de ce texte présente les premiers éléments de notre démonstration. Certains secteurs de la psychoneuro-immunologie, les principaux paramètres immunologiques évalués dans ce type d'étude, ainsi que le concept stress-émotion sont présentés. Pour de plus amples détails concernant le domaine de recherche de la PNI, le lecteur dispose de plusieurs ouvrages de référence (Ader, 1981; Ader, Felten, et Cohen, 1991; 
Herbert et Cohen, 1993; Fillion, Kirouac, Lemyre et Mandeville, 1994; KiecoltGlaser, 1988, 1992; O'Leary, 1990; Weisse, 1992).

La psychoneuro-immunologie. La PNI constitue un secteur de recherche interdisciplinaire qui s'intéresse aux relations entre les systèmes nerveux, endocrinien et immunitaire (Ader et al., 1991). Un de ces objets d'étude concerne les processus biologiques liant le stress psychologique et l'immunité (Ader, 198I). Entre le stress et la santé, les études épidémiologiques, sociologiques ou psychologiques ont indiqué des relations temporelles entre l'occurrence de stresseurs et celle de maladies (Cohen, 1985; Delongis, Folkman \& Lazarus, 1988; Miller, 1988; Minter \& Patterson, 1978; Zegans, 1982). Pour expliquer le rôle des stresseurs psychosociaux sur la santé, la thèse de la $\mathrm{PNI}$ invoque notamment une diminution de la résistance de l'hôte, se manifestant par une altération du fonctionnement immunitaire (Jemmott \& Locke, 1984). La recherche dans ce secteur a connu un essor considérable au cours de la dernière décennie. Les travaux réalisés, brièvement discutés ultérieurement, ont permis d'établir un lien entre l'altération, fonctionnelle et quantitative, de l'immunité, lors de l'exposition à un stresseur aigu (un deuil, un divorce, un examen académique) ou de l'exposition à un stresseur chronique (le chômage ou le fardeau de soin associé à une maladie chronique d'un proche). Des études récentes ont également démontré la présence de modulation de ces indices immunitaires, lors d'exposition stressogène en laboratoire.

Pour expliquer cet impact immunitaire lors de stress psychosocial et afin de fournir une base biologique permettant de lier le stress et la maladie, la $\mathrm{PNI}$ propose un mécanisme d'action immunomodulateur, lié à la réponse neurohormonale de stress. Cette réponse de stress correspond principalement à l'activation du système sympatique-adrénomédullaire (SAM), à l'activation du système hypothalamopituitaire-adrénocortical (HPAC), ainsi qu'à l'activation d'autres systèmes endocriniens (Asterita, 1985; Dantzer et Kelley, 1989; Schneiderman et McCabe, 1989; Selye, 1950). Les deux principales composantes de la réponse de stress, SAM et HPAC, représentent également une part importante de plusieurs réponses émotionnelles spécifiques (Baum, Grunberg et Singer, 1992; Sinha, Lovallo et Parsons, 1992). Cette réponse neurohormonale est décrite comme un mécanisme physiologique médiateur entre les stresseurs, le stress psychologique et la maladie (Antoni, 1987). Ces deux types de réponses ont été abondamment étudiées et notamment associées à plusieurs 
modulations immunitaires concomitantes (Ader et al., 1991; Blalock et Smith, 1985; Dantzer et Kelley, 1989; Daruna et Morgan, 1990). De plus, des aspects neurophysiologiques, reliant directement les systèmes immunitaire et nerveux, proposent une communication réciproque entre ces deux systèmes (Ader et al., 199I; Livnat, Felten, Bellinger \& Felten, 1985 ).

Le système immunitaire. Le système immunitaire (SI) joue un rôle de défense et protège l'organisme humain contre les micro-organismes générateur d'infections ou d'autres substances susceptibles de perturber l'état de santé. Le SI coordonne un ensemble de cellules et d'activité diverses visant la neutralisation et l'élimination des antigènes (voir Roitt, 1992). D'une part, les lymphocytes B qui circulent dans le sang assurent l'immunité dite «humorale» par la production d'anticorps (dont sont les immunoglobulines $\lg A$, IgD, lgE, lgG, $\lg M$ ) ayant la capacité de détruire spécifiquement l'antigène. D'autre part, les lymphocytes $T$ assurent l'immunité dite «cellulaire» en produisant des cytokines (médiateurs solubles ayant une action pharmacologique) qui permettent la régulation de la réponse immunologique ainsi que la destruction spécifique de l'antigène. Selon leur fonction, les lymphocytes $T$ se subdivisent en deux groupes: ( 1 ) les cellules $T$ «helper» $(C D 4+)$ qui influencent de façon positive la réponse immunologique, et (2) les CD8+, cellules T qui induisent des fonctions cytotoxiques ou suppressives. Par ailleurs, les réponses immunologiques non-spécifiques sont effectuées, entre autres, par les cellules NK (Natural Killer) qui reconnaissent les altérations membraneuses de certaines cellules tumorales ou de cellules infectées par des virus. Par leur capacité cytotoxique, elles jouent un rôle dans la défense contre les cancers et les infections virales.

Mesures de la réponse du système immunitaire. Parmi toutes les composantes du SI, les indicateurs immunologiques les plus fréquemment utilisés en PNI se regroupent en deux catégories, soit les mesures

192 quantitatives et les mesures fonctionnelles. Parmi les mesures quantitatives se retrouvent: (I) le nombre (décompte) des lymphocytes T helper (CD4+) et des lymphocytes $T$ suppresseurs ou cytotoxiques (CD8+), (2) le ratio CD4/CD8, (3) la quantification des cellules NK (CDI6+, CD56+ ou CD57+), (4) le dosage des anticorps totaux (ou immunoglobulines totales), (5) le dosage d'anticorps spécifiques et, (6) la quantification de cytokines, telle I'Interleukine2 (IL-2). À partir d'échantillons de sang frais provenant de la circulation périphérique, les mesures quantitatives sont généralement effectuées in vitro et correspondent au décompte des différentes sous- 
-populations de cellules effectives du SI. Parmi les mesures fonctionnelles se retrouvent: (a) la blastogenèse, transformation des lymphocytes, en réponse à une stimulation non spécifique par des agents mitogènes telle la concavaline $\mathrm{A}$ (ConA) et la phytohémagglutine ( $\mathrm{PHA}$ ) qui permettent d'évaluer l'ampleur de la division cellulaire des cellules T, ou tel le pokeweed (PKW) qui sert à l'évaluation des cellules $T$ et des cellules $B$ en division, (b) la transformation des lymphocytes en réponse à une stimulation spécifique par des antigènes tel un fragment peptidique du virus d'Epstein Barr (EBV) ou celui de l'herpès simplex de type I (HSV-I), (c) l'évaluation de l'habileté des lymphocytes à synthétiser et à libérer des cytokines, ou (d) l'estimation de l'activité cytotoxique des cellules NK (ACNK) par la libération («relargage») du chrome radioactif de cellules cibles pré-marquées qui sont détruites. Lors de la blastogenèse, il est également possible d'effectuer un dosage de cytokines telle l'IL-2. L'estimation de la réponse blastogénique ainsi que celle de la sécrétion des cytokines témoignent de l'ampleur de la réponse du SI (Fillion, Belles-Isles, Lemyre et Roy, 1994).

Les mesures quantitatives permettent la quantification du nombre de cellules effectives du SI, les mesures fonctionnelles permettent l'évaluation de leur capacité à effectuer leur fonction de défense. La variété et la complexité de ces mesures ne représentent qu'une portion simplifiée de la réponse du SI (Kiecolt-Glaser, 1988). Dans une section ultérieure où seront présentées différentes études réalisées en $\mathrm{PNI}$, la sensibilité de ces différents paramètres sera discutée. Avant de présenter ces études, l'élargissement du construit de stress est introduit en respectant la proposition de rapprocher les études sur le stress psychologique et celles portant plus spécifiquement sur les émotions (Lazarus, 1991; Scherer, 1990).

Le concept stress - émotion. La définition du stress psychologique ne fait pas consensus au sein des écrits scientifiques. Le champ du stress psychologique est vaste et comporte plusieurs volets. Parmi les plus citées, la définition du stress psychologique, qui provient du modèle transactionnel proposé par Lazarus (1966, 1991, 1993) et ses collègues (Lazarus \& Folkman, 1984; Lazarus \& Launier, 1978), se centre sur la notion de «transaction» entre les demandes contextuelles de la situation et les ressources de la personne. Le stress est vécu, lorsque l'individu évalue son bien-être menacé et ses ressources insuffisantes. Par son caractère non spécifique, le concept de stress psychologique réfère généralement à un état de détresse global (Baum, Grinberg, et Singer, 1992), et correspond souvent au pendant 
psychologique de l'activation physiologique (Ursin, 1982). Le construit de stress psychologique se distingue essentiellement de celui de l'émotion, par sa durée et son intensité (Scherer, 1990). Conceptualisant le stress comme une expérience émotionnelle, Scherer (1990) suggère l'utilisation du concept stress-émotion. Selon cet auteur, le stress est défini comme une expérience émotionnelle prolongée et souvent qualifiée d'une intensité supérieure, variant selon les caractéristiques de l'événement ou selon les les composantes spécifiques de l'évaluation personnelle. Cette conceptualisation amène également Scherer à proposer le regroupement des études portant sur les expériences de stress au sein du domaine de la pychologie des émotions. D'une façon parallèle, et sans rejeter l'existence d'un construit unidimensionnel et global de détresse associée à un stresseur psychosocial, les récents écrits de Lazarus (1991, 1993) proposent d'orienter la recherche actuelle sur le stress, vers l'étude spécifique des émotions concomitantes.

Dans le but de soutenir la thèse d'une relation entre l'émotion et l'immunité, nous retenons la suggestion de regrouper ces deux différents champs d'études, celui du stress et celui de l'émotion, par l'utilisation du concept de stress-émotion, associé globalement à un état de détresse ou spécifiquement à une émotion négative. Ayant sommairement présenté le construit stress-émotion ainsi que les mesures du SI pouvant y être associé, considérons maintenant les résultats de certains travaux empiriques permettant de soutenir la thèse d'une relation entre le stress-émotion et l'immunité.

\section{Immunité et stress-émotion: démonstration empirique}

Cette seconde section présente sommairement quelques travaux réalisés en $\mathrm{PNI}$ qui ont permis l'établissement d'un lien, entre l'exposition à un stresseur et une modulation de l'immunité (Ader et al., 1991). Le modèle théorique, souvent adopté dans ces travaux, propose que l'exposition à un stresseur soit accompagnée d'un état de détresse (stress-émotion), lui-même lié à des modulations immunitaires (Herbert et Cohen, 1993). Un sommaire d'études réalisées en milieu naturel et un résumé d'études récemment effectuées en laboratoire sont respectivement présentés. La majorité des études relèvent de la psychologie sociale; quelques-unes des études réalisées en laboratoire appartiennent directement de la psychologie des émotions. 
Les stresseurs impliquant une séparation. Les premiers travaux réalisés en PNI portent sur le deuil et sur l'état dépressif souvent associé à cet événement de vie (Bartrop, Lazarus, Luckhurst et Kiloh, 1977; Irwin, Daniels, et al., 1987, 1988; Shleifer et al., 1983). Ces études impliquent généralement un devis longitudinal, parfois de type quasi-expérimental, constitué à la fois d'un groupe endeuillé et d'un groupe de comparaison. Les sujets sont parfois appariés sur une ou plusieurs caractéristiques socio-démographiques. Au niveau immunitaire, les données font ressortir la sensibilité de l'évaluation fonctionnelle des lymphocytes $\mathrm{T}$ (en réponse à la $\mathrm{PHA}$ et à la ConA) et celle de l'activité cytotoxique des cellules NK (ACNK). Ces types de réponse fonctionnelle du SI se voient diminués dans les semaines qui suivent l'événement. De plus, ces résultats correspondent aux effets immunossuppresseurs observés lors d'études animales, réalisées auprès de jeunes singes séparés de leur mère (Coe et al., 1988). Dans certaines études, l'exposition à ce type de stresseur est également associée à une élévation de cortisol (Irwin et al., 1987; Coe et al., 1988), suggérant ainsi une relation entre le fonctionnement du SI et les états dépressifs. Les résultats de l'ensemble de ces travaux appuient l'effet immunosuppresseur de la séparation. Toutefois, l'effet n'est pas présent chez tous les sujets exposés. La diminution de l'immunité est parfois restreinte aux sujets présentant un état dépressif supérieur.

II n'est d'ailleurs pas étonnant que la recherche ultérieure dans ce secteur s'éloigne de la recherche sur le stress, et s'oriente vers l'étude clinique des patients dépressifs (Heisel, Locke, Kraus, et Williams, 1986; Kronfol et House, 1989). Tout comme dans les études sur le stress, l'indice immunitaire le plus relié aux états dépressifs est à nouveau la blastogenèse à différents mitogènes (PHA, ConA, PKW). Toutefois l'altération de l'immunité lors de dépression n'est pas présente chez tous les sujets déprimés. Les résultats provenant de ces études cliniques sont parfois même incohérents. Certains chercheurs ne distinguent pas les sujets dépressifs des non-dépressifs par leur réponse immunitaire (Schleifer, Keller, Bond, Cohen et Stein 1989). D'autres obtiennent des résultats contradictoires avec les hypothèses d'immunosuppression associée à un état dépressif: les sujets dépressifs ayant une réponse à la ConA faible présentent un état moins sévère que les sujets ayant une réponse immunitaire normale (Darko, Wilson, Gillin, et Golshan, 1991). En discutant des relations entre la sérotonine, les catécholamines et la réponse immunitaire, il est suggéré (Udelman et 
Udelman, 1991), que l'incohérence des résultats chez les patients dépressifs peut être associée aux perturbations neuroendocriniennes spécifiques de cette maladie. Dans une analyse critique de la dépression et l'immunité, Weisse (1992) signale également l'impact de la maladie sur l'immunité, et précise que les patients dépressifs présentant une diminution de la réponse blastogénique sont généralement de type unipolaire. Tout en faisant ressortir l'importance de se restreindre aux états dépressifs non cliniques, ou aux états cliniques de type unipolaire, l'ensemble des travaux soutient la thèse d'une relation entre le deuil, l'état dépressif et l'immunité.

Les examens académiques et l'immunité. Un autre stresseur naturel, dont l'occurrence est facilement contrôlable ou prévisible, concerne les examens académiques. Cette situation constitue une transaction fréquemment étudiée en $\mathrm{PNI}$, et bénéficie d'une validité écologique reconnue. Utilisant des devis de recherche de type quasi-expérimental pré-test post-test, l'ensemble de ces études atteste d'un effet immunomodulateur lors d'une situation d'examens (Fillion et al., 1989; Glaser et al., 1986, 1990, 1993; Halvorsen, et Vassend, 1987; Kiecolt-Glaser et al., 1986, Workman et Lavia, 1987). Ces travaux démontrent la sensibilité des paramètres fonctionnels suivants: la blastogenèse en réponse à la ConA et à la PHA, le dosage de l'IL-2 et l'évaluation de l'activité cellulaire des NK (ACNK); ainsi que la sensibilité des indices quantitatifs suivant: le titrage d'anticorps spécifique au EBV, le nombre de cellules NK et de cellules CD8+. Ces études relient des stresseurs aigus à l'immunité, et appuient l'hypothèse d'un effet immunodépresseur associé au stress. Toutefois, l'immunomodulation n'est pas toujours présente et semble à nouveau liée à la réponse de stress-émotion. Afin d'expliquer ces variations individuelles, des caractéristiques psychologiques, incluant l'évaluation cognitive et les stratégies de gestion, sont proposées comme médiateur (Herbert et Cohen 1993; Pelletier \& Herzing, 1989; Vingerhoests \& Assies, 1991). Des résultats appuient l'importance des modes de gestion. La relaxation peut en effet atténuer les effets immunodépresseurs (Kiecolt-Glaser et al., 1986), alors que l'intrusion cognitive (Workman et Lavia, 1987) semble l'amplifier. Bien que proposée dans plusieurs discussions, l'évaluation cognitive n'est que partiellement, ou pas mesurée. Par ailleurs, l'effet immunomodulateur semble également varier selon la durée et la temporalité de la transaction, de même que selon le type de paramètre immunologique étudié (Fillion, Kirouac et al., 1994). Le manque de standardisation dans le moment des prises de mesures rend 
difficile la comparaison des résultats. L'étude de stresseurs chroniques peut contourner certains de ces problèmes.

Les stresseurs chroniques et l'immunité. Les stresseurs chroniques réfèrent à des situations perdurantes. L'infertilité, (Kedem, Bartoov, Mikulincer et Shkolnik, 1992), ta situation de fardeau familial lié à la maladie d'Alzheimer, (Kiecolt-Glaser et al., 1987), ainsi que le chômage (Arnetz et al., 1987, 1991) ont été associés à des diminutions graduelles de la réponse immunitaire. Malgré les faiblesses méthodologiques associées principalement aux devis corrélationnels, ces études appuient l'hypothèse d'immunodépression lors de stress. L' ensemble de ces résultats, ajoutés à ceux provenant des études réalisées lors d'exposition à des stresseurs naturels, aigus ou chroniques, soutiennent l'hypothèse d'un lien entre les états de stress-émotion et le fonctionnement du SI. Des différences individuelles sont toutefois observées. Parfois la modulation immunitaire n'est pas liée à la condition d'exposition, et est davantage associée à l'intensité de la réponse de stress-émotion. Pour expliquer cette différence d'intensité, l'inclusion de variables intermédiaires, tels l'évaluation cognitive et le mode de gestion, est proposée. Ainsi, pour qu'un stresseur affecte le fonctionnement du SI, les premières études réalisées en $\mathrm{PNI}$ amènent à formuler l'hypothèse conditionnelle de la prévalence d'un état de stress-émotion intense et prolongé.

Les stresseurs en laboratoire et l'immunité. Afin de vérifier cette dernière hypothèse ainsi que la présence de lien de causalité entre les stresseurs et l'immunité, des études en laboratoires sont réalisées. Suite à l'exposition à un stresseur, une réactivité immunitaire, quantitative et fonctionnelle, est observée (Bachen et al., 1992; Manuck et al., 1991; Naliboff et al., 1991). À peine quelques minutes suivant l'exposition au stresseur, les sujets manifestent une augmentation du nombre de cellules CD8 +, une élévation du nombre ainsi que de la fonction des cellules NK, et une diminution de la blastogenèse aux mitogènes ConA et PHA. La réaction observée est de fait très comparable à celle suivant une injection d'épinéphrine et fait ressortir le rôle prépondérant du système nerveux sympathique (SNS) (Kiecolt-Glaser et al., 1992), quoique l'amplitude de cette réactivité demeure associée à la sécrétion de cortisol (Cacioppo, 1994). Par ailleurs, malgré un patron cohérent d'effets immunitaires, des différences individuelles demeurent observables. Certains sujets réagissent très fortement. Les sujets qui manifestent une réponse importante au niveau immunitaire manifestent également une réactivité cardiovasculaire accrue (Manuck et al., 1991; Sgoutas et al., 1992; Stone et al., 1993; Zakowski et al., 1992, 1994). II devient 
de plus en plus évident que l'exposition à un stresseur affecte la réactivité immunitaire et la réponse cardiovasculaire de façon concomitante. Dans le domaine de recherche cardiovasculaire, l'étude des différences individuelles, présentée ultérieurement, fait également ressortir l'importance des variables psychologiques médiatrices.

Les études sur les émotions et l'immunité. Considérant que l'émotion est souvent conceptualisée comme un bref phénomène (Ekman, 1984), et considérant la rapidité de la réactivité immunitaire décrite dans les études sur le stress, la recherche liant l'immunité et l'émotion devient de plus en plus pertinente. Quoique peu nombreuses, l'induction d'émotion en situation de laboratoire a permis de réaliser que les émotions négatives ou positives sont associées à un profil comparable de modulations immunitaires fonctionnelles (Futterman et al., 1994; Knapp et al. 1992). L'amplitude de la réactivité immunitaire est plus prononcée lors d'émotion négative (Knapp et al. 1992) et est associée aux indices cardio-vasculaires d'activation du SNS (Futterman et al., 1994). Ces études sont cohérentes avec les études réalisées sur le stress et font ressortir que la réactivité immunitaire est également associée à l'induction d'émotions positives.

La recherche réalisée en laboratoire permet de lier la réponse neurohormonale de stress et le SI. De plus concernant I'hypothèse conditionnelle d'un état émotif intense et prolongé, la rapidité de la réactivité immunitaire tend à infirmer la notion de nécessité d'un caractère soutenu, justifiant en ce sens la recherche liant l'émotion et l'immunité. Toutefois l'association entre la réactivité immunitaire et l'activation du SNS appuie la condition d'intensité: les sujets hautement réactifs au niveau cardio-vasculaire manifestent également une réactivité immunitaire supérieure. La recherche en laboratoire sur l'émotion et l'immunité permet de préciser que l'amplitude de la réactivité cardiovasculaire est plus importante que la valence positive

198 ou négative de l'émotion. Par ailleurs, tout comme la recherche en milieu naturel, la recherche en laboratoire fait ressortir d'importantes différences individuelles dans les profils de réactivité immunitaire.

La première section de ce texte a permis la précision du construit de stress-émotion et la présentation sommaire des mesures du SI pouvant être associées. La seconde section a présenté des travaux empiriques permettant de soutenir la thèse d'une relation entre le stress-émotion et l'immunité. Cette dernière section propose l'inclusion de l'évaluation cognitive pour expliquer une part des différences individuelles observées. 


\section{Différences individuelles et évaluation cognitive}

Selon la perspective transactionnelle (Lazarus et Launier, 1978; Lazarus et Folkman, 1984), l'intensité et l'amplitude de la réponse de stress-émotion dépendent: $a$ ) de l'évaluation cognitive de l'enjeu de la transaction (potentiel menaçant ou dommageable du stresseur; première composante de l'évaluation cognitive), ainsi que $b$ ) de l'évaluation de la capacité à prévenir ou à gérer cette situation (seconde composante). Malgré un consensus sur l'importance d'inclure un médiateur cognitif dans les recherches sur le stress, le construit et la définition opérationnelle de l'évaluation cognitive demeurent à préciser (Monroe et Kelley, 1995).

Dans le domaine de la recherche sur le stress, l'évaluation cognitive a souvent été mesurée par l'intermédiaire d'un seul item portant sur la perception immédiate de l'individu (menace, défi, perte ou bénéfice) spécifique à un stresseur donné (Moos et Schaefer, 1993). Ce type de mesure est principalement utilisé dans les études réalisées en laboratoire et présente l'avantage d'évaluer directement et spécifiquement le construit en accord avec le modèle transactionnel. Malgré la pertinence de l'information donnée par ce type évaluation, le format restreint à un seul item peut présenter des limites psychométriques. De plus, l'information sur la validité, la stabilité et les caractéristiques spécifiques de ce type de mesure demeure restreinte (Monroe et Kelley, 1995). Pour évaluer le construit d'évaluation cognitive, une autre stratégie est proposée avec la recherche sur le stress, axée sur la mesure des événements de vie. Dans ce secteur de la recherche, le stress est associé au nombre (Holmes et Rahe, 1967) ainsi qu'aux caractéristiques contextuelles (Brown et Harris, 1978) des événements de vie ou des stresseurs. L'inventaire des événements de vie est généralement réalisé, soit par l'intermédiaire d'un questionnaire, ou soit lors d'une entrevue structurée. Afin de considérer les différences individuelles lors de l'estimation du nombre de stresseurs, l'inclusion de dimensions subjectives s'est graduellement ajoutée. Avec ce type de mesure, l'évaluation cognitive des dimensions d'impact (Horowitz et al., 1979; Sarason et al., 1978), de désirabilité (Rhodewalt et Zone, 1989; Vinokur et Selzer, 1975), d'importance (Parkes, 1986), de contrôle (Averill, 1973; Flannery, 1986; Folkman, 1984; Thompson, 1981), de menace (Lazarus, 1966; Mason, 1975), de prévisibilité, d'incertitude et d'anticipation (Paterson et Neufeld, 1987) a, tour à tour, été étudiée à titre de variables médiatrices, dans la relation 
entre la personne et l'événement stressant. Certaines de ces mesures permettent de distinguer les deux composantes de l'évaluation cognitive proposées par le modèle transactionnel de stress, d'autres pas. Afin de mieux représenter le construit, et d'en extraire les principales composantes, l'évaluation cognitive est parfois mesurée en regroupant plusieurs dimensions. Ainsi, Holm et al. (1986) identifient trois composantes, qui correspondent respectivement à l'Impact, le Contrôle et la Prédictibilité. Lemyre et ses collaborateurs $(1986,1993)$ identifient également un modèle incluant deux ou trois composantes: I'Impact, la Maîtrise et parfois, l'Incertitude. La variance de ce dernier facteur semble restreinte à certains stresseurs aigus (Lemyre et Tessier, 1995), variant possiblement selon le statut de résolution de l'événement; en d'autres circonstances, la contribution des items associés à ce facteur, demeure associée aux composantes Impact et Maîtrise (Fillion, 1993). Globalement, ces auteurs proposent de regrouper plusieurs dimensions de l'évaluation cognitive d'un stresseur afin d'identifier les composantes principales. Cette stratégie empirique permet l'identification d'au moins deux facteurs qui se rapprochent théoriquement du modèle transactionnel de stress et qui se dessine comme une avenue intéressante pour définir opérationnellement le construit. Bien que les démarches de validation de ce construit se poursuivent dans le domaine du stress, les récents travaux de Lazarus (Lazarus, 1991; Smith et Lazarus, 1993) proposent d'élargir le construit en précisant d'une part, le contenu des composantes, et d'autre part, la spécificité de ce contenu en fonction de différentes émotions.

Parallèlement aux travaux sur le stress, la précision du contenu et de la spécificité des composantes de l'évaluation cognitive constitue un objet d'étude en psychologie de l'émotion (Roseman, Spindel et Jose, 1990; Wallbott et Scherer, 1991). Dans le domaine des émotions, plusieurs théories cognitives ont été proposées afin d'élaborer une définition opérationnelle du construit de l'évaluation cognitive, applicable pour toutes les émotions (Arnold, 1960; Roseman, 1984, Smith et Ellsworth, 1985; Scherer, 1988). Pour définir ce construit, Arnold (1960) propose trois dimensions: conséquences (positive ou négative), contrôle (capacité à gérer) et occurrence (temporalité: est-ce présent en ce moment?). Roseman et al. (1984) en proposent cinq: enjeux de la situation, possibilité de contrôle, probabilité, lieu de contrôle et intérêt ou motivation; Scherer (1988) en propose également cinq: agrément, potentiel de maîtrise, nouveauté, rapport aux buts et accord avec les standards; enfin, Smith et al. $(1985,1989)$ en propose six: agrément, 
contrôle situationnel, incertitude, lieu de contrôle, attention et effort anticipé. Malgré certaines limites psychométriques des échelles utilisées pour mesurer ces dimensions de l'évaluation, des convergences entre ces théories permettent de soutenir la validité de convergence du construit. En comparant, au niveau de 16 émotions, le pouvoir prédictif de trois modèles nommés ci-haut (Arnold, Roseman et Scherer), Roseman et al. (1990) identifient des facteurs de convergence. Concernant des émotions facilement associables à l'état de stress, telles la détresse et la peur, les auteurs font ressortir les facteurs cognitifs suivants: les enjeux, le potentiel de maîtrise, l'occurrence, l'attribution et la motivation. Notons que les trois premiers facteurs sont facilement comparables aux composantes proposées en psychologie sociale. Tout en chevauchant théoriquement les deux principales composantes de l'évaluation cognitive proposées dans les modèles de stress, la définition opérationnelle de l'évaluation cognitive, au sein des théories de l'émotion, offre un potentiel accru de dimensions et propose un patron spécifique d'organisation de celles-ci, selon l'émotion. L'évolution respective de ces deux domaines de recherche, le stress et l'émotion, pourrait éventuellement déboucher sur la définition opérationnelle d'un seul construit d'évaluation cognitive. Ce construit unique inclurait un nombre exhaustif d'items permettant de représenter à la fois les deux composantes du modèle transactionnel, et à la fois le regroupement spécifique de différentes dimensions.

L'évaluation cognitive et l'immunité. Au sein de plusieurs théories, se retrouve l'inclusion d'un médiateur cognitif entre l'exposition à un stresseur et la réponse de stress. Selon le type de stresseur et notamment, selon la possibilité de contrôle perçu, un mode de gestion actif ou passif (inhibé) est déployé (Schneiderman et McCabe, 1989). Chacun de ces modes de réponse semble accompagné d'une modification hémodynamique et endocrinienne caractéristique. Au mode actif, correspondent notamment une augmentation du rythme cardiaque, une élévation de la tension artérielle systolique, une sécrétion de catécholamines et une dominance de la composante du SAM. Le mode passif ou inhibé se caractérise davantage par une réponse hémodynamique vasculaire, c'est-à-dire par une augmentation de la résistance périphérique des vaisseaux, et est également accompagné d'une sécrétion de corticostéroïdes témoignant de la composante HPAC (Schneiderman et McCabe 1989). Un profil mixte est également possible (Schneiderman et McCabe, 1989). Les émotions de peur 
et de joie semblent principalement associées à la composante SAM alors que l'émotion de colère apparait simultanément liée aux deux composantes (Sinha, Lovallo et Parsons, 1992). Dépendamment des théories, ce mode de gestion correspond à différentes appellations: «effort ou détresse» (Frankenhaueser, 1980), «lutte/fuite ou retrait» (Cannon, 1930), «combat actif ou défaite» (Folkow, 1993). Malgré l'importance accordée à l'évaluation cognitive au sein de de ces modèles, leur démonstration a été principalement réalisée par des études animales, excluant ainsi la possibilité de mesurer directement le construit. Même dans les études humaines, les recherches ont davantage porté sur les caractéristiques du stresseur ou les conditions contextuelles de l'expérimentation (contrôle, prévisibilité, compétition, harcèlement, consignes) pouvant potentiellement moduler l'évaluation cognitive et affecter la réponse neurohormonale de stress (Lazarus et Folkman, 1984). Si la recherche a permis de vérifier l'association entre certains types de stresseurs et le mode de réponse (Hurwitz et al., 1993; Zakowski et al., 1994), les dimensions cognitives n'ont toutefois que rarement, partiellement ou indirectement été évaluées (Monroe et Kelley, 1995). Ainsi malgré son rôle théorique prépondérant pour expliquer l'occurrence de différents profils de stress, l'hypothèse du rôle médiateur de l'évaluation cognitive ne dispose que d'appuis indirects, et demeure à vérifier.

Pour illustrer la pertinence d'inclure l'évaluation cognitive dans les études en $\mathrm{PNI}$, nous terminons par la présentation de nos travaux, réalisé à la fois en milieu naturel, lors d'un diagnostic de cancer (Fillion et al., 1993, 1995, 1996) et en laboratoire (Fillion, 1995).

Évaluation cognitive et immunité lors de d'un diagnostic de cancer. L'étude est effectuée auprès de 72 femmes rencontrées avant et après une chirurgie diagnostique. Suite au diagnostic de cancer du sein de stade précoce, 36 femmes sont appariées, sur 4 variables socio-démographiques, à 36 femmes recevant un diagnostic de tumeur bénigne. Les résultats suggèrent que la période d'anticipation du diagnostic est associée à un plus grand niveau de perturbation que celle suivant le diagnostic de cancer comme tel. Les patientes qui perçoivent plus d'Incertitude et associent davantage d'Impact au fait d'avoir le cancer semblent être celles qui manifestent les niveaux de stress les plus élevés, et les patientes qui présentent un meilleur sentiment de Maîtrise face au cancer et une perception pré-post cohérente concernant l'Impact de cette maladie semblent présentées une meilleure immunité fonctionnelle (PHA et ConA) et quantitative (NK). Les résultats confirment 
l'importance de l'évaluation cognitive du diagnostic pour expliquer les différences individuelles observées lors de l'adaptation psychoimmunologique au cancer du sein de ce stade.

Évaluation cognitive et immunité lors d'un stresseur en laboratoire. Cette seconde étude, réalisée cette fois en laboratoire, porte sur le rôle médiateur de l'évaluation cognitive, lors d'une exposition à un bref stresseur. Un groupe de sujets séropositifs au $\mathrm{VIH}(\mathrm{VIH}+)$, non diagnostiqué SIDA, est comparé à un groupe de sujets non infectés par le virus $(\mathrm{VIH}-)$. Le stresseur choisi consiste à préparer, ainsi qu'à livrer un exposé oral. Lors de cet exposé, le sujet est invité à se défendre verbalement contre une fausse accusation de vol à l'étalage. Des instructions guident la préparation ainsi que la présentation de l'exposé. Des travaux antérieurs ont permis d'observer une association entre la période d'exposition (préparation/exposé) et le mode de réponse: la période de préparation étant associée à un mode de gestion actif, alors que la période d'exposé verbal étant liée un mode mixte (Hurwitz et al., 1993). Le stresseur choisi permet ainsi l'occurrence potentielle des deux modes de réponses. Les mesures cardiovasculaires et immunitaires sont prises avant l'exposition, en période de préparation, en période de présentation et en période de récupération. La mesure cognitive est administrée immédiatement après la période de récupération, avant le retrait des électrodes, du brassard et du cathéter veineux. Pour l'ensemble des sujets, une association négative entre la perception de Maîtrise et la réactivité physiologique est observée. En ce qui concerne la réactivité immunitaire, plusieurs indices de la réactivité immunitaire quantitative (augmentation du nombre de leucocytes et de lymphocytes totaux, et du nombre absolu de cellules $\mathrm{CD} 8+$ ) et fonctionnelle (augmentation de l'activité cytotoxique des cellules NK(ACNK) ) sont négativement corrélés avec la perception de Maîtrise. Un profil différent, voire même opposé, est observé selon le groupe, en ce qui concerne la relation entre la perception d'Impact et la réactivité. Chez le groupe HIV+, plusieurs indices de la réactivité cardiovasculaire et immunitaire quantitative (augmentation du nombre de leucocytes et de lymphocytes totaux, et du nombre absolu de cellules CD4+) se retrouvent négativement associés à la perception d'Impact. Les résultats confirment la présence d'associations significatives entre l'évaluation cognitive et la réponse de réactivité physiologique. Les résultats observés chez l'ensemble des sujets appuient les modèles théoriques voulant qu'une perception de contrôle (Maîtrise) affecte le mode de réactivité quoique l'observation attentive des 
résultats observés chez les sujets $\mathrm{VIH}+$ laisse envisager un rôle médiateur moins important à la perception de contrôle et fait davantage ressortir l'importance de la perception d'Impact négatif. Concernant cette dimension de l'évaluation cognitive (Impact), les relations observées chez les sujets de ce groupe $(\mathrm{VIH}+)$, présentent une direction opposée à ce qui devrait théoriquement être observé. Plus le sujet infecté perçoit la situation menaçante, moins il semble réagir au stresseur, alors que l'opposé est observé chez le groupe $\mathrm{VIH}$-. [Les résultats sont discutés en fonction de la théorie du syndrome d'adaptation et en fonction de l'évolution de l'infection $\mathrm{au} \mathrm{VlH}$ ]. Ces résultats s'ajoutent à ceux de l'étude précédente et confirment la contribution significative de l'évaluation cognitive dans l'explication des différences individuelles observées dans les profils de réactivité immunitaire.

\section{CONCLUSION}

En respectant une proposition de rapprocher les études sur le stress et celles portant plus spécifiquement sur les émotions, une intégration de différentes recherches réalisées en PNI a permis, dans un premier temps, de soutenir la thèse d'une relation entre l'exposition à un événement stressogène, un phénomène émotionnel et une altération du fonctionnement immunitaire. Alors que les premières études réalisées en PNI proposaient initialement une immunosuppression conditionnelle à la prévalence d'un état de stress-émotion intense et prolongé, les études réalisées en laboratoire confirment la notion d'intensité et infirment la notion de durée, justifiant ainsi la recherche liant l'émotion et l'immunité. L'association entre la réactivité immunitaire et l'activation du SNS appuie d'autant plus la condition d'intensité: les sujets hautement réactifs au niveau cardiovasculaire manifestent également une réactivité immunitaire supérieure. La recherche en laboratoire sur l'émotion et l'immunité permet de préciser que l'amplitude de la réactivité cardiovasculaire est plus importante que la valence positive ou négative de l'émotion. Par ailleurs, tout comme la recherche en milieu naturel, la recherche en laboratoire fait ressortir d'importantes différences individuelles dans les profils de réactivité immunitaire. Pour expliciter ces différences, l'intégration de ces travaux fait ressortir, dans un second temps, la nécessité d'inclure des variables médiatrices. Enfin la présentation de deux études confirme la contribution significative de l'évaluation cognitive dans 
l'explication des différences individuelles observées dans les profils de réactivité immunitaire. Par ailleurs, l'intégration de la recherche sur le stress et l'émotion permet de définir le stress psychologique selon une perspective globale (détresse) et spécifique (émotion). De la même façon, cette intégration encourage les efforts de recherche sur la définition opérationnelle d'un seul construit d'évaluation cognitive favorisant une utilisation globale ou spécifique. La nécessité de circonscrire le construit de stress se justifie par le besoin fréquent de restriction du nombre de variables dans certains protocoles de recherche. La possibilité d'élargir ce construit global par l'évaluation spécifique constitue assurément un ajout lorsque la description des profils de réaction nécessite plus de précision. L'ensemble de ce travail appuie la proposition de fusionner les deux domaines de recherche et encourage fortement l'inclusion d'un volet immunologique dans les études sur l'émotion.

\section{RÉFÉRENCES}

Ader, R. (1981). Psychoneuroimmunology. New York: Academic Press.

Ader, R., Felten, D.L., \& Cohen, N. (1991) Psychoneuroimmunology. (2nd ed.) New York: Academic Press.

Antoni, M. H. (1987). neuroendocrine influences in psychoimmunoology and neoplasia. Psychology anfd Health, 1, 3-24.

Arnetz, B.B., Brenner, S.O., Levi, L., Hjelm,R., Petterson, I.L., Wasserman, J., Petrini, B., Eneroth, P., Kallner, A., Kvetnansky, R., \& Vigas, M. (199I). Neuroendocrine and immunologic effects of unemployment and job insecurity. Psychotherapy Psychosomatic, 55, 76-80.

Arnetz, B.B., Wasserman, J., Petrini,B., Brenner, S.O., Levi,L., Eneroth, P., Salovaara, H., Hjelm, R., Salovaara, L., Theorell, T., \& Petterson, I.L. (1987). Immune function in unemployed woman. Psychosomatic Medicine, 49, 3-13.

Arnold, M. B. (1960). Emotion and Personality: Vol. I. Psychological aspects. N.Y.: Columbia University Presss.

Asterita, M. F. (1985). The physiology of stress. New York : Human Sciences Press.

Averill, J. R. (1973). Personal control over aversive stimuli and its relationship to stress. Psychological Bulletin, 80, 286-303.

Bachen, E.A., Manuck , S.B., Marsland, A.L.,Cohen, S., Malkoff,S.B., Muldoon, M.F., \& Rabin, B.S. (1992). Lymphocyte subset and cellular immune responses to a brief experimental stressor. Psychosomatic Medicine, 54, 673-679.

Bartrop, R.W., Lazarus, L., Luckhurst, E., \& Kiloh, L.G. (1977). Depressed lymphocytes function after bereavement. Lancet, I, 834-836.

Baum, A., Grunberg,N.E., \& Singer, J.E. (1992). Biochemical measurements in the study of emotion. Psychological Science, 3, 56-59. 
Blalock, J.E., \& Smith E.M. (1985). A complete regulatory loop between the immune system and neuroendocrine systems. Federation Proceedings, 44, 108-III.

Brown, G.W., \& Harris, T. (1978). Social Origins of Depression. New-York: Free Press.

Cacioppo, J.T. (1994). Social neuroscience: Autonomic, neuroendocrine, and immune responses to stress. Psychophysiology, 31, 113-128.

Cannon, W. (1931). Studies on the conditions of activity in the endocrine organs. American Journal of Physiology, 98, 447-452.

Coe, C., Rosenberg, L., \& Levine, V. (1988). Prolonged effect of psychological disturbance on the squirrel monkey. Brain, Behavior and Immunity, 2, 151-160.

Cohen, F. (1985). Stress and bodily illness. Dans A. Monat \& R.S. Lazarus (Eds.), Stress and coping (pp.40-54). N.Y. : Columbia Press University.

Dantzer, R., \& Kelley, K.W. (1989). Stress and immunity: integrated view of relationships between brain \& immune system. Life-Sciences, 44, 1995-2008.

Darko, D. F., Wilson, N.W., Gillin, J. C., \& Golshan, S. (|99|). A critical appraisal of mitogen-induced lymphocytes proliferation in depressed patients. American Journal of Psychiatry, 148, 337-344.

Daruna, J. H., \& Morgan, J. E. (1990). Psychosocial effects on immune function: neuroendocrine pathways. Psychosomatics, 31, 4-12.

Delongis, A., Coyne, J. C., Dakof, G., Folkman, S., \& Lazarus, R. S. (1982). Relationship of daily hassles, uplifts, and major life events to health status. Health Psychology, 1, I 19$-136$.

Ekman, P. (1984). Expression and the nature of emotion. Dans K.S. Scherer, \& P.Ekman (EDS) Approaches to emotion (319-343) Hillsdale, NJ:Erlbaum.

Felten, D. (199|). Neurotransmetter signalling of cells of the immune system: Important progress, major gaps. Brain, Behavior and Immunity, 5, 2-8.

Fillion, L. (1995). Cognitive appraisal, HIV status and immune reactivity. Dans L. Bessette (Ed.), Le deuil comme processus de guérison (pp. 126-128), Beauport: Publication $\mathrm{MNH}$.

Fillion, L. (1993). Évaluation cognitive, état de stress et immunité lors d'un diagnostic de tumeur au sein. Thèse de doctorat non-publiée. Québec: Université Laval.

Fillion, L., Belles-Isles, M., Lemyre, L., \& Roy, R. (1994). Reliability of lymphocyte proliferation assays. Stress Medicine, 10 ( 1 ).

Fillion, L., Kirouac, G., Lemyre, L., \& Mandeville, R. (1994). Stresseur et immunité.: une recension en psychoneuroimmunologie. Psychologie Canadienne, 35, 405-426.

Fillion, L., Lemyre, L., Mandeville, R., \& Piché, R. (1996). Facteurs cognitifs lors de l'adaptation psycho-immunologique au diagnostic de cancer du sein: implication clinique. Journal de Thérapie Comportementale et Cognitive, 6, 43-5I.

Fillion, L., Lemyre, L., Piché, R., \& Mandeville, R. (1996). Cognitive appraisal, stress state and cellular immunity before and after breast tumor diagnosis. International Journal of Rehabilitation and Health, 2, 169-187.

Fillion, L., Lemyre, L., Mandeville, R., Piché, R. Poisson, R., Fournelle-Lebuis, F. \& Falardeau, M. (1993). Cellular Immunity and psychological stress before and after diagnostic surgery of breast cancer. Clinical and Investigative Medicine, 16 (3).

Fillion, L., Tessier, R., Tawadros, E., \& Mouton, C. (1989). Stress et immunité: étude de validité d'une mesure de stress psychologique (MSP). Psychologie Canadienne, 30, 30-38.

Flannery, R. B. (1986). Personal control as a moderator variable of life stress: preliminary inquiry. Psychological Reports, 58, 200-202. 
Folkman, S. (1984). Personal control and stress and coping process: a theoretical analysis. Journal of Personality and Social Psychology, 46, 839-852.

Forsythe, C.J., \& Compas, B.E. (1987). Interaction of cognitive appraisals of stressful events and coping: Testing the goodness-of-fit hypothesis. Cognitive Therapy and Research, I I, 473-485.

Folkow, B. (1993). Psysiological organization of neurohormonal responses to psychosocial stimuli: implications for health and disease. Annals of Behavioral Medicine, 15, 236-244.

Futterman, A.D., Kemeny,M.E., Shapiro, D., \& Fahey, J.L. (1994). Immunological and physiological changes associated with induced positive and negative mood. Psychosomatic medicine, 56 : 499-511.

Frankenhaeuser, M. (1980). Psychoneuroendocrine approaches to the study of stressful person-environment transactions. Dans H. Selye (Ed)(pp.46-70). Selye's guide to stress research. N.Y.: Van. Nortrand Reinhold Company.

Friedman, E. S., Clark, D. B., Gershon, S. (1992). Stress, anxiety and depression : Review of Biological, diagnostic, and nosologic issues. Journal of Anxiety Disorders, 6, 337-363.

Glaser, R., Kennedy, S., Lafuse, W. P., Bonneau, R. H., Speicher, C., Hilhouse, J., \& Kiecolt-Glaser, J. K. (1990). Psychological stress induced modulation of interleukin 2 receptor gene expression and interleukin 2 production in peripheral blood leukocytes. Archives of General Psychiatry, 47, 707-719.

Glaser, R., Pearson, G.R., Bonneau, R.H., Esterling, B.A., Atkinson, C., \& Kiecolt-Glaser, J. (1993). Stress and the memory T-cell response to the Epstein-Barr virus in healthy medical students. Health Psychology, 12, 435-442.

Glaser, R., Rice, J., Speicher, C. E., Stout, J., \& Kiecolt-Glaser, J. K. (1986). Stress depresses interferon production by leukocytes concomitant with a decrease in N.K.C.A. Behavioral Neuroscience. 100, (5), 675-678.

Halvorsen, R., \& Vassend, O. (1987). Effects of examination stress on some cellular immunity function. Journal of Psychosomatic Research. 31, 693-701.

Heisel, J.S., Locke, S.E., Kraus, L.J., \& Williams, R.M. (1986). Natural Killer cell activity (NKCA) and MMPI scores of a cohort of college students. American Journal of Psychiatry, 143, 1382 $-1384$.

Herbert, T.B., \& Cohen, S. (1993). Stress and immunity in humans : A meta-analytic review. Psychosomatic Medicine, 55, 364-379.

Holm, J.E., Holroyd, K. A., Hursey, K. G., \& Penzien, D.B. (1986). The role of stress in Recurrent Tension Headache. Headache, 26, 160-167.

Holmes, T. H., \& Rahe, R. H. (1967). The social readjustment rating scale. Journal of Psychosomatic Research, 11, 213-218.

Horowitz, M.J., Wilner, N., \& Alvarez, W. (1979). Impact of event scale: A measure of subjective stress. Psychosomatic Medicine, 4I, 209-218.

Hurwitz, B.E., Nelesen, R.A., Saab, P.G., Nagel, J.H., Spitzer, S., Gellman, M., McCabe, P., Phillips, D.J., \& Schneiderman, N. (1993). Differential patterns of dynamic cardiovascular regulation as a function of task. Biological Psychology, 36, 75-95.

Irwin, M., Daniels, M., Risch, S. C., Bloom, E., \& Weinwe, H. (1988). Plasma cortisol and naturel killer cell activity (NKCA) during bereavement. Biological Psychiatry, 24, 173-178.

Irwin, M., Daniels, M., \& Weiner, H. (1987). Immune and neuroendocrine change during bereavement. Psychiatric Clinics North America, 10, 449-465.

Jemmott , J. B., \& Locke, S.E. (1984). Psychosocial factors, immunologic mediation and human susceptibility to infectious disease: How much do we know? Psychological Bulletin, 95, 78-108. 
Kedem, P., Bartoov, B., Mikulincer, M., \& Shkolnik, T. (1992). Psychoneuroimmunology and male infertility : a possible link between stress, coping and male immunological infertility. Psychology and Health, 6, 159-173.

Kiecolt-Glaser, J. K., \& Glaser, R. (1992). J. of Consulting and Clinical Psychology, 60, I-7.

Kiecolt-Glaser, J. K. (1988). Methodological issues in behavioral immunology research with humans. Brain, Behavior \& Immunity, 2, 67-78.

Kiecolt-Glaser, J. K., Galser, R., Strain, E., Stout, J., Tarr, K. L., Holliday, J. E., \& Speicher, C.E. (1986). Modulation of cellular immunity in medical students. Journal of Behavioral Medicine, 9, 5-21.

Kiecolt-Glaser, J. K., \& Glaser, R. (1988). Psychological influences on immunity. American Psychologist, 43, 892-898.

Kiecolt-Glaser, J.K., Glaser, R., Shuttleworth, E.C., Dyer, C.S., Ogrocki, P. \& Speicher, C.E. (1987b). Chronic stress and immunity in family caregivers of Alzheimer's disease victims. Psychosomatic Medicine. 49, (5), 523-535.

Knapp, P.H., Levy, E. M., Giorgi, R. G., Black, P. H., Fox, B. H., \& Heeren, T. C. (1992). Short-term immunological effects of induced emotion. Psychosomatic Medicine, 54, I33-148

Kronfol, Z., \& House, J.D. (1989). Lymphocyte mitogenis, immunoglobulin and complement levels in depressed patiens and normal controls. Acta Psychiatrica Scandinavia, 80, 142-147.

Lazarus, R.S. (1993). From psychological stresss to emotions: a history of changing outlooks. Annual Review of Psychology, 44, 1-21.

Lazarus, R. S. (199|). Theory based stress measurement. Psychological Inquiry, I, 4I-5I.

Lazarus, R. S. (1966). Psychological stress and the coping process. N.Y.: Mc-Graw Hill.

Lazarus, R. \& Folkman, S. (1984). Stress, appraisal and coping. N.Y.: Springer Pub. Company.

Lazarus, R. S., \& Launier, R. (1978). Stress-related transactions between person and environment.. In L.A. Pervin et M. Lewis (Eds). Interactional Psychology. NY. : Plenum Press.

Lemyre, L., \& Tessier, R. (in preparation). Stressor appraisal rating scales across methodologies: Perceived impact, mastery and uncertainty.

Lemyre, L. (1986). Stress et appréhension cognitive. Thèse non publiée. Univ. Laval, Québec.

Livnat, S., Felten, S.,Y., Bellinger, D. L., \& Felten, D. (1985). Involvement of peripherical and central catecholamine systems in neural-immune interactions. J.of Neuroimmunology, 10 , 5-30.

Manuck, S. B., Cohen , S., Rabin, B. S., Muldoon, M. F. \& Bachen, E. A. (1991). Individual differences in cellular immune response to stress. Psychological Science, 2, I| I-1 I5.

Mason, J.W. (1975). Emotion as reflected in patterns of endocrine integration. In L. Levi (Ed.), Emotions: Their parameters and measurement (pp. 143-181). N.Y. : Raven Press.

McNaughton, M. E., Smith, L.W., \& Grant, I. (1990). Stress, social support, coping resources, and immune status in elderly women. Journal of Nervous and Mental Disease, 178, 460-461 .

Miller, T. (1988). Advances in understanding the impact of stressful life events on health. Hospital and Community Psychiatry, 39, 6, 615-622.

Minter, R. E., \& Patterson, C. (1978). Life events and illness onset: a review. Psychosomatics, 19, 334-339.

Monroe, S.M., \& Kelley, J.M. (1995). Measurement of stress appraisal. Dans S. Cohen, R.C. kessler, \& L.U.Gordon (Eds), (122-147). Measuring Stress. N.Y.: Oxford Univesity Press.

Moos, R.H., \& Schaefer, S.C. (1993). Coping ressources and processes: Current concepts and measures. Dans L. Goldberger \& S. Breznitz (Eds.) (234-257). Handbook of stress (2nd edition). N.Y.: The Free Press. 
Naliboff, B. D., Benton, D., Solomon, G. F., Morley, J. E., Fahey, J. L., Bloom, E.T., Makidodan, T., \& Gilmore, S. L. (199I). Immunological changes in young and old adults during brief laboratory stress. Psychosomatic Medicine, 53, 121-132.

O'Leary, A. (1990). Stress, emotion and human immune function. Psychological Bulletin, 108, 363-382.

Parkes, K. R. (1986). Coping in stressful episodes: the role of individual differences, environmental factors and situational characteristics. Journal of Personnality and Social Psychology, 51, 1277-1292.

Paterson, R. J., \& Neufeld, R.W.J. (1987). Clear danger: Situational determinants of the appraisal of threat. Psychological Bulletin, 101, 404-416.

Paykel, E. S. (1987). Methodology of life events research. Advances in Psychosomatic Medicine, 17. 13-29.

Pelletier, R., \& Herzing, D. L. (1989). Psychoneuroimmunolgy: Toward a mind body model: A critical review. Advances, 5, 27-56.

Roitt, I. (1992). Essential Immunology (7ème Ed.) London: Blackwell.

Roseman, I. J. (1984). Cognitive determinants of emotions : A structural theory. Dans P. Shaver (Ed.) Review of Personality and social psychology (1 I-36). Bevely Hills, CA: Sage.

Roseman, I. J., Spindel, M. S., \& Jose, P. E. (1990). Appraisal of emotion eliciting events: Testing a theory of discrete emotions. J. of Personality and Social Psychology, 59, 899-925.

Sarason, I. G., Johnson, J. H., \& Siegel, J. M. (1978). Assessing the impact of life changes: Development of the Life Experience Survey. J. Consulting and Clinical Psychology, 46, 932-946.

Scherer, K. R. (1988). Criteria for emotion-antecedent appraisal: A review. Dans V. Halminton, G.H. Bower, \& N.H.Frijda (Eds.). Cognitive perpectives on emotion and motivation (pp. 89 -126). Norwell, MA: Kluwer Academic.

Scherer, K. R. (1990). Stress et coping: nouvelles approches. Cahiers Psychiatriques Genevois, $9,147-154$.

Schleifer, S. J., Keller, S. E., Bond, R. N., Cohen, J., \& Stein, M. (1989). Major depressive disorder and immunity. Archives of General Psychiatry, 46, 81-87.

Schleifer, S. J., Keller, S. E., Camerino, M., Thornton, J. C., \& Stein, M. (1983). Suppression of lymphocyte stimulation following bereavement. JAMA, 250, 374-377.

Schneiderman, N., \& McCabe, P. (1989). Psychophysiologic strategies in laboratory research. In N. Schneiderman, S.M. Weiss, \& P.G. Kaufman (Eds.), Handbook of research methods in cardiovascular behavioral medicine (pp349-364). N.Y.: Plenum.

Selye, H. (1950). The physiology and pathology of exposure to stress. Montréal: Acta.

Sgoutas-Emch, S.A., Cacioppo, J.T., Uchino, B.N., Malarkey, W., Pearl, D., Kiecolt-Glaser, J., \& Glaser, R. (1994). The effects of an acute psychological stressor on cardiovascular endocrine, and cellular immune response: A prospective study of individuals high and low in heart rate reactivity. Psychophysiology, 31, 264-27l.

Sinha, R., Lovallo, W.R., \& Parsons, O.A. (1992). Cardiovascular differentiation of emotions. Psychosomatic Medicine, 54, 422-435.

Smith, C.A. (1989). Dimensions of Appraisal and physiological response in emotion. Journal of Personality and Social Psychology, 56, 339-353.

Smith, C.A., \& Ellsworth, P. C. (1985). Patterns of Cognitive Appraisal in emotion. Journal of Personality and Social Psychology, 48, 813-838.

Smith,C.A., \& Lazarus, R.S. (1993). Appraisal components, core relational themes, and the emotions. Cognition \& Emotion, 7, 233-269. 
Stone, A.A., Valdimarsdottir, H.B., Katkin, E.S., Burns, J., \& Cox, D.S. (1993). Effects of mental stressor on mitogen-induced lymphocyte responses in the laboratory. Psychology and Health, 8, 269-284.

Syvalahti, E. (1987). Endocrine and immune adaptation in stress. Annals of Clinical Research, 19, 70-77.

Thompson, S. C. (198I). Will it hurt less if I can control it ? A complex answer to a simple question. Psychological Bulletin, 90, 89-101.

Udelman, D. L., \& Udelman, H. D. (199|). Affects, neurotransmitters and immuno-competence. Stress Medicine, 7, 159- 162.

Ursin, H. (1982). The search for stress markers. Scandinavian J.of Psychology, Supp, I65-169.

Vinokur, A., \& Selzer, M. L. (1975). Desirable versus undesirable life events: their relationship to stress and mental distress. Journal of Personality and Social Psychology. 32, 329-337.

Vingerhoests, A. J. J. M., \& Assies, J. (199|). Psychoneuroendocrinology of stress and emotions: Issues for future research. Psychotherapy Psychosomatic , 55, 69-75.

Watson, D., \& Pennebaker, J.W. (1989). Health complaints, stress, and distress: Exploring the central role of negative affectivity. Psychological Review, 96, 234-254.

Walbott, H. G., \& Scherer, K. R. ( 199 |). Stress specificities: Differential effects of copyng style, gender, and type of stressor on autonomic arousal, facial expression and subjective feeling. Journal of Personality and Social Psychology, 61, 147-156.

Weisse, C. S. (1992). Depression and immunocompetence: A review of the literature. Psychological Bulletin, 111, 475-489.

Weisse, C. S., Pato, C. N., McAllister, C.G., Littman, R., Breier, A., Paul, S.M., \& Baum, A. (1990). Differential effects of controllable and uncontrollable acute stress on lymphocyte proliferation and leukocyte percentages in humans. Brain, Behavior \& Immunity, 4, 339-35I.

Workman, E. A ., \& LaVia, M.F. (1987). T-lymphocyte polyclonal proliferation and stress response style. Psychological Reports, 60, $1121-1122$.

Zautra, A. J., Okun, M. A., Robinson, S. E., Lee, D., Roth, S.H., \& Emmanual, J. (1988). Life stress and lymphocites alterations among patiens with rheumatoid arthritis. Health Psychology, $8,1-14$.

Zakowski, S.G.,Cohen L., hall, M.H., Wollman, K., \& Baum, A. (1992). Differential effects of active and passive laboratory stressors on immune function in healthy man. International Journal of behavioral Medicine, I, 163-184.

Zakowski, S.G., McAllister, C. G., Deal, M. , \& Baum, A. (1992). Stress, Reactivity, and Immune Function in Healthy Men. Health Psychology, 11, 223-232.

Zegans, L.S. (1982). Stress and the development of somatic disorders. In L. Goldberg \& S. Breznitz (Eds), Handbook of stress ( Pp |34- 152). N.Y. : Free Press. 
(Página deixada propositadamente em branco) 
Série

Investigaçāo

Coimbra

Imprensa da Universidade

Les Presses de L'Université Laval

Les Editions de L'IORC

2004 